\title{
Dynamics of Computational Islet Simulations: Islets with majority mutated open $\mathrm{K}_{\mathrm{ATP}}$ channels retain bursting
}

\author{
Gemma Gearhart ${ }^{1}$, Shuai Jiang ${ }^{2}$, Thomas J. May ${ }^{3}$, Jane Pan ${ }^{4}$, \\ Samuel Khuvis ${ }^{4}$, Matthias K. Gobbert ${ }^{4,}{ }^{*}$, Bradford E. Peercy ${ }^{4}$, \\ Arthur Sherman ${ }^{5}$
}

\begin{abstract}
The study of pancreatic beta-cells comprises a crucial part of the study of the group of diseases known as diabetes. These cells exist in groups known as islets of Langerhans and are responsible for storing and producing insulin. They exhibit electrical bursting behavior during insulin production that correlates with the rate at which insulin is secreted into the bloodstream. Coupling is a natural process within islets that enables the cells to communicate with one another and transfer various ions and electrical currents; coupling of both voltage and metabolites can occur. We model multicellular islets using an existing system of seven ordinary differential equations to model beta cell function. We simulate cells with mutated $\mathrm{K}_{\mathrm{ATP}}$ channels that remain open indefinitely, which have been described in experimental studies but not yet modeled. Simulations ran with these mutations reveal the existence of a bursting death threshold, described by the least percentage of cells in the islet that must be mutated for electrical bursts to completely disappear. We determine that this threshold is independent of coupling strengths, cell distribution, and possibly islet dimension; however, we also determined that this threshold is dependent on the glucose influx rate.
\end{abstract}

Keywords: beta cell model, computational islet, glycolytic oscillations

\section{Introduction}

Diabetes mellitus, commonly known as diabetes, is a group of diseases in which the body either does not have the capabilities of producing insulin or is not capable of producing sufficient amounts of insulin to compensate for the glucose that enters the body. According to the American Diabetes Association (ADA), this set of diseases affects the lives of approximately $8.3 \%$ of the United States population of all ages and racial backgrounds [6]. However its prevalence is scattered across all regions of the world. Normally, when blood sugar levels are increased by the consumption of glucose, a healthy human body would naturally produce adequate amounts of insulin to remove excess glucose that could potentially be harmful if maintained. The biological units that are responsible for the secretion of insulin are identified as beta-cells [14], which are one of four hormone-producing cells located

\footnotetext{
${ }^{1}$ Division of Science, Mathematics and Computing, Bard College at Simon's Rock

${ }^{2}$ Department of Mathematics, Cornell University

${ }^{3}$ Department of Mathematics, Virginia Polytechnic Institute and State University

${ }^{4}$ Department of Mathematics and Statistics, University of Maryland, Baltimore County

${ }^{5}$ Laboratory of Biological Modeling, National Institutes of Health

${ }^{*}$ Correspondence: gobbert@umbc.edu
} 


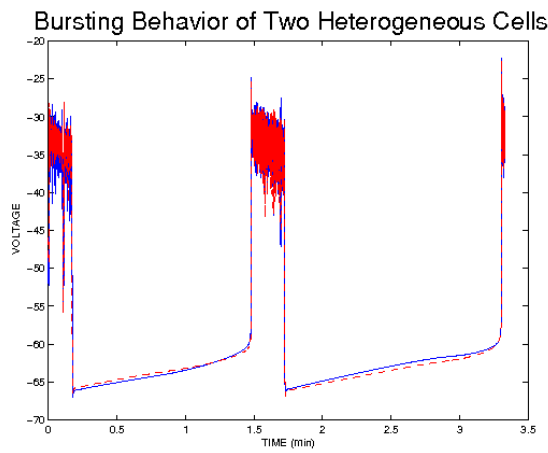

(a)

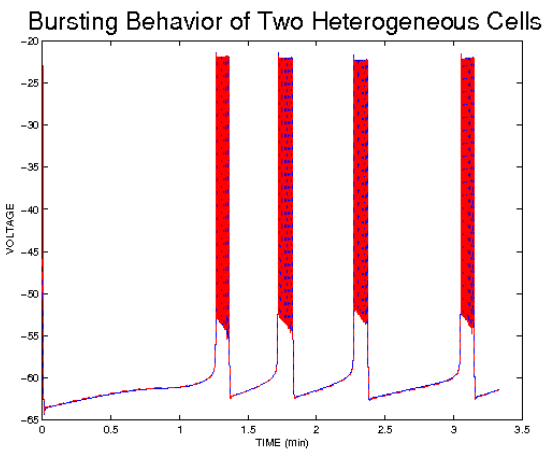

(b)

Figure 1: (a) Oscillation behavior of two coupled beta-cells with an electrical coupling value of $0.01 \mathrm{pS}$. (b) Oscillation behavior of the beta-cells with an electrical coupling value of $0.1 \mathrm{pS}$.

in the pancreas. Clusters of beta-cells are known as islets of Langerhans, which depend on other islets within the pancreas to successfully carry out the production of insulin.

While Type I Diabetes involves the immune system directly attacking the beta-cells, Type II Diabetes is caused by the increasing resistance that the body has towards the effects of insulin rather than the lack of beta-cells directly. In order to have a better understanding of diabetes in the hopes of finding effective methods to treat it, it is necessary to analyze the behavioral patterns exhibited by pancreatic beta-cells.

Previous studies have observed that beta-cells undergo voltage oscillations during the course of insulin secretion. Once glucose enters the beta-cell, the cell itself experiences an increase in ATP, which leads to the depolarization of the beta-cell, allowing calcium to enter and insulin to be released into the bloodstream [10]. The frequency at which insulin is secreted correlates to the oscillating behavior of the cells. Oscillations are grouped in bursts of electrical activity.

Coupling is a natural process that occurs between cells within the islets of Langerhans. Gap junctions serve as pathways for the coupling that occurs between these cells, allowing for effective communication from cell to cell, including the transfer of ionic currents and calcium ions [1]. Previous findings have supported the notion that certain variations of coupling play a vital role in facilitating the production of insulin [14]. This behavior can be seen primarily in the bursting patterns that the cells experience.

Figure 1 displays the oscillation behaviors of two heterogeneous beta-cells when they are electrically coupled with one another. Both plots display continuous voltage alterations between rapid spiking behavior and stages of gradual increase. The rapid voltage oscillations denote the bursting action of the beta-cells, signifying the release of insulin. The smooth lines following these rapid spiking phases represent the periods of rest before insulin is secreted again.

Cases in which the cells contain stronger electrical coupling strengths eventually become synchronized. Figure 1 contains the results that are obtained when the electrical coupling strength is increased by a factor of 10 . It can be observed that the oscillations between the two cells are more harmonized, which suggests that the bursting behaviors between the two heterogeneous cells are similar.

Recent experiments (Benninger et al. [2], Nguyen et al. [11]) show that mutations in a potassium channel (KATP) that reduces sensitivity to ATP:ADP ratios as well as trafficking to the beta-cell membrane can impact the influx of calcium and the resultant secretion of insulin. The method used to impart this mutation was a transgene that alters the functional KATP channels but not in all of the beta-cells in an islet. Furthermore, the electrical coupling strength between the beta-cells of an islet can coordinate otherwise heterogeneous 
beta-cells. Both Benninger et al. and Nguyen et al. reduce coupling in an islet and unmask heterogeneity in KATP channel mutated islets by releasing mutated or unmutated cells to burst depending on closed or open form of the KATP channel mutation. We have developed a computational islet [10] that we use to mutate mathematically KATP channels in a fraction of islet cells. Here we observe the level of open KATP channel mutation necessary for loss of islet bursting.

\section{Mathematical Model}

To approach this problem, we represent a computational islet using an $N \times N \times N$ lattice of beta-cells, where $N$ represents the dimensions of the lattice measured in number of cells.

One of the main focuses of our project was using these models to study the factors that provoke oscillation deaths. The term "death" is used to signify the loss in slow metabolic oscillations that the beta-cells exhibit. We looked at cases with distributions containing a certain proportion of mutated cells, in which the $\mathrm{K}_{\mathrm{ATP}}$ channels of the cells are always open. Similar to what we did for the first part of our research, we observe the bursting behaviors that result from the addition of these mutated cells. We also determine the specific proportion of mutated channels that serve as a threshold between oscillating patterns and oscillation deaths. Specific distributions of these mutated cells can be found in Section 3.1.

After running tests that focused on manipulating the coupling strengths and varying the proportions of cells with mutated $\mathrm{K}_{\mathrm{ATP}}$ channels, we were able to gain a clearer understanding on how these parameters play a role in the oscillating behavior of the cells. When determining the types of initial conditions that caused metabolic oscillations in beta-cells, we found that certain combinations of coupling at specific ranges of coupling strengths cause gradual reduction in metabolic oscillations. Furthermore, we noticed that having greater than $74 \%$ mutated cells in the distribution causes metabolic oscillation death regardless of the conditions of the other parameters. Details on specific inputs and results we obtained can be found in Section 4. Final conclusions are made in Section 5.

Oscillations in voltage and metabolites, such as glucose 6-phosphate (G6P) and fructose 1-6-bisphosphate (FBP), occur when glucose enters beta-cells through special protein channels in the cell membrane called glucose transporters (e.g., GluT1 or GluT2) via facilitated diffusion. This only occurs when the concentration of glucose outside the beta-cell is high. The cell then metabolizes the glucose in the mitochondria and produces adenosine triphosphate (ATP). Increases in the concentration of ATP triggers depolarization of betacell membranes by closing channels of ATP-sensitive $\left(\mathrm{K}_{\mathrm{ATP}}\right)$ channels, which causes calcium to enter the cell. The subsequent influx of calcium and increase in $[\mathrm{Ca}]$ causes the release of vesicles containing insulin into the blood stream. Glucose levels are then decreased due to the release of insulin and the beta-cell resets itself [9]. The reaction rate of glucokinase (called $R_{\mathrm{GK}}$ ) reflects the level of glucose stimulation and does not change during the course of a simulation.

The $\mathrm{K}_{\mathrm{ATP}}$ channels that facilitate membrane depolarization are the main regulator of electrical activity in the islet, and glucose-stimulated insulin secretion relies on the sensitivity of these channels to ATP-inhibition. Mutations that render $\mathrm{K}_{\mathrm{ATP}}$ channels insensitive to ATP-inhibition suppress glucose-stimulated insulin secretion and alter voltage levels in the islet. Mutations of these channels can occur such that they remain either open or closed.

In each cell, the beta-cell model uses glycolytic dynamics initially developed by Smolen in [12], combined with electrical dynamics by Bertram et al. [3], and studied by TsanevaAtanasova et al. [14]. The model's dependent variables are voltage $V$, fraction of open potassium channels $n$, and five other chemical concentrations. The equations form a stiff 
system of seven coupled ordinary differential equations (ODEs). The equations are

$$
\begin{aligned}
\frac{d V}{d t} & =-\frac{I_{\mathrm{K}}+I_{\mathrm{Ca}}+I_{\mathrm{K}(\mathrm{Ca})}+I_{\mathrm{K}(\mathrm{ATP})}}{C_{m}} \\
\frac{d n}{d t} & =\frac{n_{\infty}-n}{\tau_{n}}, \\
\frac{d[\mathrm{Ca}]}{d t} & =f_{\mathrm{cyt}}\left(J_{\mathrm{mem}}+J_{\mathrm{er}}\right), \\
\frac{d[\mathrm{Ca} \mathrm{er}]}{d t} & =-\sigma_{V} f_{\mathrm{er}} J_{\mathrm{er}}, \\
\frac{d[\mathrm{ADP}]}{d t} & =\frac{[\mathrm{ATP}]-[\mathrm{ADP}] \exp \left\{(r+\gamma)\left(1-\frac{[\mathrm{Ca}]}{r_{1}}\right)\right\}}{\tau_{a}}, \\
\frac{d[\mathrm{G} 6 \mathrm{P}]}{d t} & =k\left(R_{\mathrm{GK}}-R_{\mathrm{PFK}}\right), \\
\frac{d[\mathrm{FBP}]}{d t} & =k\left(R_{\mathrm{PFK}}-0.5 R_{\mathrm{GPDH}}\right),
\end{aligned}
$$

where $[\mathrm{XX}]$ denotes the concentration of $\mathrm{XX}$ in the cytosol for calcium (Ca), Adenosine diphosphate (ADP), glucose-6-phosphate (G6P), fructose-1,6-bisphosphate (FBP), and in the endoplasmic reticulum (ER) for calcium $\left(\mathrm{Ca}_{\mathrm{er}}\right)$. The forms for consumption of $\mathrm{G} 6 \mathrm{P}$ due to phosphofructose kinase $R_{\mathrm{PFK}}$ and dehydrogenase consumption of FBP $R_{\mathrm{GPDH}}$ can be found in [3].

Additional definitions are

$$
\begin{aligned}
I_{\mathrm{K}} & =\bar{g}_{K} n\left(V-V_{\mathrm{K}}\right), \\
I_{\mathrm{Ca}} & =\bar{g}_{\mathrm{Ca}} m_{\infty}(V)\left(V-V_{\mathrm{Ca}}\right), \\
I_{\mathrm{K}(\mathrm{Ca})} & =g_{\mathrm{K}(\mathrm{Ca})}\left(V-V_{\mathrm{K}}\right), \\
g_{\mathrm{K}(\mathrm{Ca})}([\mathrm{Ca}]) & =\frac{\bar{g}_{\mathrm{K}(\mathrm{Ca})}([\mathrm{Ca}])}{1+\left(\frac{K_{d}}{[\mathrm{Ca}]}\right)^{2}}, \\
I_{\mathrm{K}(\mathrm{ATP})} & =g_{\mathrm{KATP}}\left(V-V_{\mathrm{K}}\right), \\
g_{\mathrm{KATP}} & =\bar{g}_{\mathrm{K}, \mathrm{ATP}} o_{\infty}([\mathrm{ADP}]), \\
m_{\infty}(V) & =\frac{1}{1+\exp \left[-\frac{20+V}{12}\right]}, \\
J_{\mathrm{er}} & =0.0002\left(\left[\mathrm{Ca} a_{\mathrm{er}}\right]-[\mathrm{Ca}]\right)-0.4[\mathrm{Ca}], \\
J_{\mathrm{mem}} & =\left(4.5 \times 10^{-6}\right) I_{\mathrm{Ca}}-0.2[\mathrm{Ca}],
\end{aligned}
$$

where

$$
o_{\infty}([\mathrm{ADP}])=\frac{0.08\left(1+2 \frac{0.165[\mathrm{ADP}]}{17}\right)+0.89\left(\frac{0.165[\mathrm{ADP}]}{17}\right)^{2}}{\left(1+\frac{0.165[\mathrm{ADP}]}{17}\right)^{2}\left(1+\frac{0.135[\mathrm{ADP}]}{26}+0.05[\mathrm{ATP}]\right)} .
$$

The potassium channel has current, $I_{\mathrm{K}}$, with maximal conductance, $\bar{g}_{K}$, and gating variable $n$ driven by the potential's difference from the channel's Nernst potential, $V_{\mathrm{K}}$. Similarly, the calcium, calcium-sensitive potassium, and ATP-sensitive potassium channel with currents $I_{\mathrm{Ca}}, I_{\mathrm{K}(\mathrm{Ca})}$, and $I_{\mathrm{K}(\mathrm{ATP})}$, each have a maximal conductance $\bar{g}_{\mathrm{K}(\mathrm{Ca})}, \bar{g}_{\mathrm{Ca}}$, and $\bar{g}_{\mathrm{K}(\mathrm{ATP})}$. Each also has conductance dynamics defined by $m_{\infty}(V), \frac{[\mathrm{Ca}]}{1+\left(\frac{K d}{[\mathrm{Ca}]}\right)^{2}}$ with sensitivity parameter $K_{d}$, and $o_{\infty}([\mathrm{ADP}])$, respectively. Calcium enters the cytosol via the membrane, $J_{\text {mem }}$, or from the ER, $J_{\mathrm{er}}$. A corresponding amount is lost from the ER, $-J_{\mathrm{er}}$. Each calcium dynamic is scaled by $f_{\text {cyt }}$ or $f_{\text {er }}$ representing buffering in the respective compartment, and $\sigma_{V}$ is the ratio of cytosol to ER volumes. The parameter $k$ scales the rates of metabolism 
reactions. The factor $\exp \left\{(r+\gamma)\left(1-\frac{[\mathrm{Ca}]}{r_{1}}\right)\right\}$ captures calcium feedback onto the production of ADP produced with a time constant, $\tau_{a}$. We use constants: $C_{m}=5300 \mathrm{fF}$, $V_{\mathrm{K}}=-75 \mathrm{mV}, g_{\mathrm{K}}=2700 \mathrm{pS}, \tau_{n}=20 \mathrm{~ms}, g_{\mathrm{Ca}}=1000 \mathrm{pS}, V_{\mathrm{Ca}}=25 \mathrm{mV}$. Note that $R_{\mathrm{GK}}, \bar{g}_{\mathrm{K}(\mathrm{Ca})}$, and $\bar{g}_{\mathrm{K}, \mathrm{ATP}}$ are parameters that impact bursting rates of electrical potential (voltage). For example, in Figure 1 the fast bursting cell has $R_{\mathrm{GK}}=0.4, \bar{g}_{\mathrm{K}(\mathrm{Ca})}=25000 \mathrm{pS}$, and $\bar{g}_{\mathrm{KATP}}=600 \mathrm{pS}$, while the slow bursting cell has $R_{\mathrm{GK}}=0.2, \bar{g}_{\mathrm{KCA}}=27000 \mathrm{pS}$, and $\bar{g}_{\mathrm{KATP}}=100 \mathrm{pS}$. In the rest of the work, the cell heterogeneity is due only to changes in $o_{\infty}([\mathrm{ADP}])$.

In order to couple the cells together, we define a matrix $G$ that contains the coupling strengths between each pair of cells. The matrix $G$ will be defined such that the coupling is diffusive $\left(\alpha\left(V_{i}-V_{j}\right)\right.$ where $\alpha$ is the coupling strength, $V_{i}$ is the neighboring cell and $V_{j}$ is the current cell). Thus the differential equation can be written as

$$
\frac{d y}{d t}=f(t, y)+G y
$$

where $y=(V, n,[\mathrm{Ca}],[\mathrm{Ca} \mathrm{er}],[\mathrm{ADP}],[\mathrm{G} 6 \mathrm{P}],[\mathrm{FBP}])^{T}$ is a vector of length $7 N^{3}$ (with component vectors $V=\left[V_{1}, \ldots, V_{N^{3}}\right]^{T}$, etc. of length $\left.N^{3}\right), f(t, y)$ is the right hand side of equations (1)-(7). $G$ is a sparse block diagonal connectivity matrix with $7 \times 7$ blocks defined as

$$
G=\left(\begin{array}{cccccc}
C_{V} & 0 & \cdots & 0 & 0 & 0 \\
0 & 0 & \cdots & 0 & 0 & 0 \\
\vdots & \vdots & \ddots & \vdots & \vdots & \vdots \\
0 & 0 & \cdots & 0 & 0 & 0 \\
0 & 0 & \cdots & 0 & C_{G 6 P} & 0 \\
0 & 0 & \cdots & 0 & 0 & C_{F B P}
\end{array}\right)
$$

where the $(a, b)$ entry of the matrices $C_{V}, C_{G 6 P}, C_{F B P}$ contains the coupling strength between the $a$ th cell and the $b$ th cell, $g_{V}, g_{G 6 P}$, and $g_{F B P}$, respectively. As in [10], $a$ and $b$ are indexed with $i+(j-1) N+(k-1) N^{2}$ being the one-dimensional index of the $(i, j, k)$-th cell with $1 \leq i, j, k \leq N$. Let $\nu=\mathrm{V}, \mathrm{G} 6 \mathrm{P}$, or FBP. Then, $C_{\nu}$ is a matrix with seven non-zero bands. In our model, the coupling strength is the same throughout the islet. So, the elements on the main diagonal of $C_{\nu}$ are the number of neighbors that each cell has multiplied by -1 and the coupling strength of the species $\nu$. On the first superdiagonal and subdiagonal, $N$ superdiagonal and subdiagonal, and the $N^{2}$ superdiagonal and subdiagonal are the coupling strength of the species $\nu$.

\section{Methodology}

We now move to modifying the existing seven variable model for our study.

\subsection{Simulating Mutations}

In [2], Benninger experimentally studied the effects of mutated $\mathrm{K}_{\mathrm{ATP}}$ channels on calcium oscillations in islets of beta-cells in mice. We extend the seven variable model so that it can handle cases in which a specified portion of cells in the islet have mutated $\mathrm{K}_{\text {ATP }}$ channels that remain fully open or fully closed (see Section 2). Although our code is capable of simulating both mutation types, we only investigate the open mutation case; closed mutations are a possible direction for further study.

From [14], we know that $o_{\infty}([A D P])$ from (8) controls the open and closed behavior of $\mathrm{K}_{\mathrm{ATP}}$ channels; however, this number is not a fractional amount of open channels. In order to determine appropriate values for $o_{\infty}$ in mutated cells, we numerically solved the ODEs (1)-(7) under different coupling conditions and distributions and observed the values of $o_{\infty}$ during the run. Based on our observations, we set $o_{\infty}$ to 0.0075 for the open mutation as the largest observed values for $o_{\infty}$. 
Given these observations, we redefine $o_{\infty}([A D P])$ to

$$
o_{\infty}([A D P])= \begin{cases}0.0075 & : \text { Open Mutation, } \\ \alpha([A D P]) & : \text { Normal Cell, }\end{cases}
$$

where the steady-state open fraction from (8)

$$
\alpha([A D P])=\frac{0.08\left(1+2 \frac{0.165[\mathrm{ADP}]}{17}\right)+0.89\left(\frac{0.165[\mathrm{ADP}]}{17}\right)^{2}}{\left(1+\frac{0.165[\mathrm{ADP}]}{17}\right)^{2}\left(1+\frac{0.135[\mathrm{ADP}]}{26}+0.05[\mathrm{ATP}]\right)}
$$

now applies to normal cells.

For our investigations of islets with cells with open mutations, we are interested in two distributions with $m \leq N^{3}$ mutated cells and $N^{3}-m$ non-mutated cells:

1. Mutated Grouped: Set cells 1 to $m$ to be mutated cells and cells $m+1$ to $N^{3}$ to be non-mutated cells.

2. Mutated Spread: Set cell 1 to be mutated then set cell $N^{3}$ to be mutated. Continue placing a mutated cell halfway between mutated cells and evenly distributed until there are $m$ mutated cells (i.e., at cells $1, N^{3}, N^{3} / 2, N^{3} / 4,3 N^{3} / 4, N^{3} / 8,3 N^{3} / 8,5 N^{3} / 8$, $7 N^{3} / 8$, etc.).

For the case of open mutations, the burst death threshold is the number of mutated cells in the islet required for no voltage bursts to be observed in the first 10 minutes of the simulation. The main focus of our mutation studies is to see how this threshold value is effected by different electrical and metabolic coupling, cell distributions, and $R_{\mathrm{GK}}$ values. The different studies we run for the $5 \times 5 \times 5$ islet are as follows:

1. Electrical Coupling Study: This study varies electrical coupling strength from $25 \mathrm{pS}$ to $600 \mathrm{pS}$ incrementing by $25 \mathrm{pS}$ while holding G6P and FBP coupling constant at $0.0 \mathrm{~ms}^{-1}$ and $R_{\mathrm{GK}}$ at $0.2 \mathrm{~s}^{-1}$ for both types of distributions.

2. FBP Coupling Study: This study varies FBP coupling strength from $0 \mathrm{~ms}^{-1}$ to $0.1 \mathrm{~ms}^{-1}$ incrementing by $0.005 \mathrm{~ms}^{-1}$ while holding G6P coupling constant at $0.0 \mathrm{~ms}^{-1}$, voltage coupling at $100 \mathrm{pS}$, and $R_{\mathrm{GK}}$ at $0.2 \mathrm{~s}^{-1}$ for both types of distributions.

3. G6P Coupling Study: This study varies G6P coupling strength from $0 \mathrm{~ms}^{-1}$ to $0.1 \mathrm{~ms}^{-1}$ in increments of $0.005 \mathrm{~ms}^{-1}$ while holding FBP coupling at $0.01 \mathrm{~ms}^{-1}$, voltage coupling at $100 \mathrm{pS}$, and $R_{\mathrm{GK}}$ at $0.2 \mathrm{~s}^{-1}$ for both distributions.

4. $\mathbf{R}_{\mathrm{GK}}$ Study: This study varies $R_{\mathrm{GK}}$ from $0 \mathrm{~s}^{-1}$ to $0.6 \mathrm{~s}^{-1}$ incrementing by $0.025 \mathrm{~s}^{-1}$ while holding voltage coupling at $100 \mathrm{pS}$, FBP coupling at $0 \mathrm{~ms}^{-1}$, and G6P coupling at $0 \mathrm{~ms}^{-1}$ for both types of distributions.

We also run a sampling of these studies for the $3 \times 3 \times 3$ islet to see if and how varying islet size affects the threshold.

\subsection{Numerical Method}

Given the different reaction rates of the system of differential equations (1)-(7), we have to treat this system as a stiff system of differential equations. We use a memory-modified version of MATLAB's ode15s with automatic differentiation developed in [7] to run our simulations. MATLAB's standard implementation of ode15s uses a version of Numerical Differentiation Formulas (NDFk), which is the standard method for stiff systems of differential equations. In order to solve the system, NDF $k$ requires a Jacobian to be supplied, 
which MATLAB allows to be handled in multiple ways. We supply a sparse Jacobian derived analytically using automatic differentiation, computed via the ADiMat software [4]. This is the optimal choice for our studies because it has a significant speedup compared to most other methods, as observed in [10] and confirmed for the particular case of this paper in Section 4.2, as well as allows us to make modifications to the model in our simulations without having to recompute the Jacobian by hand.

MATLAB's standard ode15s has significant speed and memory issues in solving the seven variable model even when supplied with a Jacobian found using automatic differentiation. The memory-modified version developed in [7] reduces the amount of memory allocated for each iteration of the process and removes the feature of ode15s which stores the gradient vector for each iteration since it is not used in any of the post-processing for our model. Efficiency tests in [10] show that there is a significant speedup when the combination of ADiMat and the memory-modified ode15s is used, which allows for more simulations.

In addition, usage of MATLAB's Parallel Computing Toolbox allowed for significantly higher simulation throughput. The command parfor is a parallel for-loop for studies with multiple runs of code, one for each of the for-loop values. Each for-loop parameter typically represents one parameter value, for which a run of the code is needed. On the cluster tara with 8 computational cores on each node, MATLAB distributes the job associated with each parameter value in the parallel for-loop to one of the 8 cores. Since these jobs are independent of each other and run simultaneously, the overall study can be completed 8 times as fast as a serial run.

\subsection{Description of the Computing Environment}

The computations for this study were performed using the MATLAB programming language (www.mathworks.com) under the Linux operating system on the cluster tara, located in the UMBC High Performance Computing Facility (www. umbc.edu/hpcf). The distributedmemory cluster tara consists of 86 nodes, with each node containing two quad-core Intel Nehalem X5550 processors (2.66 GHz, $8 \mathrm{MB}$ cache) and 24 GB memory, thus up to 8 parallel processes can be run simultaneously per node. All nodes and the $160 \mathrm{~TB}$ central storage are connected by a quad-data rate InfiniBand interconnect.

\section{Results}

Using the extended capabilities of our model, we simulate and describe effects of varying parameters in a multicellular islet and extend our study to simulate islets in which some cells have mutated $\mathrm{K}_{\mathrm{ATP}}$ channels.

\subsection{Observing the Effects of Open $\mathrm{K}_{\mathrm{ATP}}$ Channel Mutations}

Our model considers cells with either of two types of $\mathrm{K}_{\mathrm{ATP}}$ channel mutations: always open, which leads to hyperpolarized and unresponsive cells, and always closed, which forces cells to be consistently bursting. We investigate only the case of open mutations but have simulation capabilities of both cases in our model. These mutations alter the sensitivity of $\mathrm{K}_{\mathrm{ATP}}$ channels to ATP-inhibition, which changes the value of the current $I_{\mathrm{K}(\mathrm{ATP})}$ and, in consequence, the cell's voltage (see (1)). Based on this structure of influence within our model, we expect that the presence of mutated cells in the islet will affect voltage oscillations most severely. We expect the degree of influence to depend on the percentage of cells in the islet with channel mutations.

Figure 2 shows a detailed study of electrical oscillation behavior as open $\mathrm{K}_{\mathrm{ATP}}$ channel mutations are introduced into a $5 \times 5 \times 5$ computational islet with an $R_{\mathrm{GK}}$ value of $0.2 \mathrm{~s}^{-1}$ in both types of cells. As the number of mutated cells increases, the number of voltage bursts observed in cells in the islet gradually decreases. Voltage bursts continue to reduce until they eventually disappear when 93 of the 125 cells, or $74 \%$, are mutated. Figure 2 shows the 

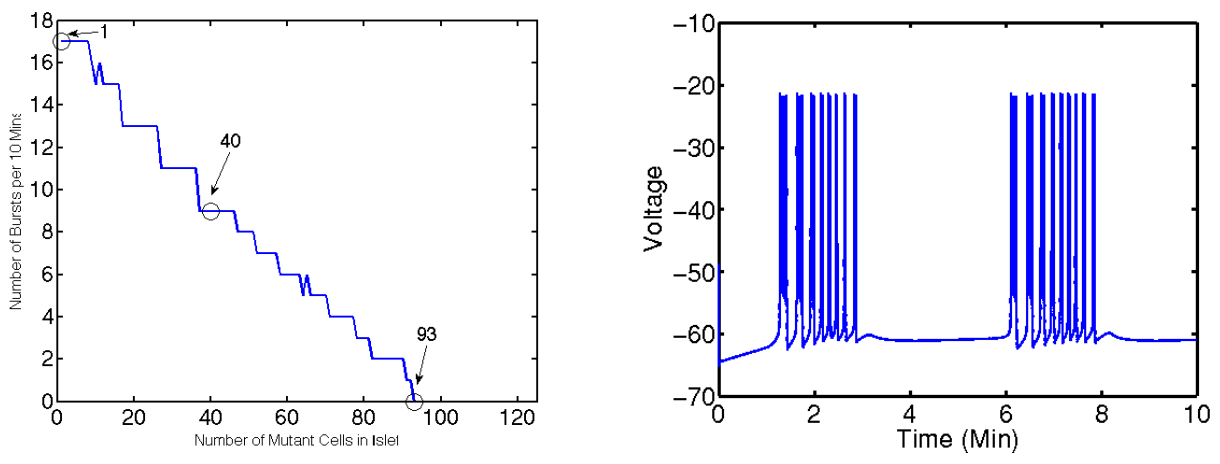

1 Mutated Cell

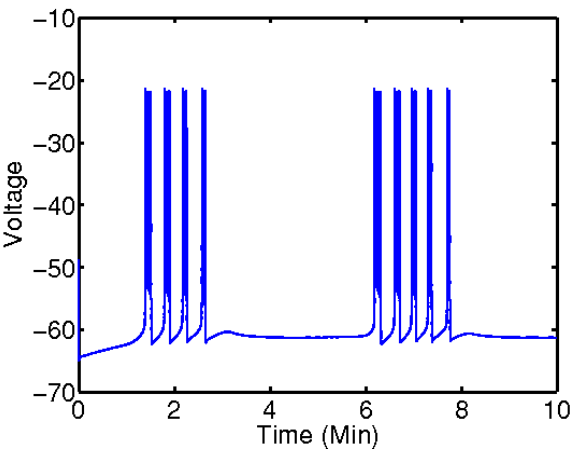

40 Mutated Cells

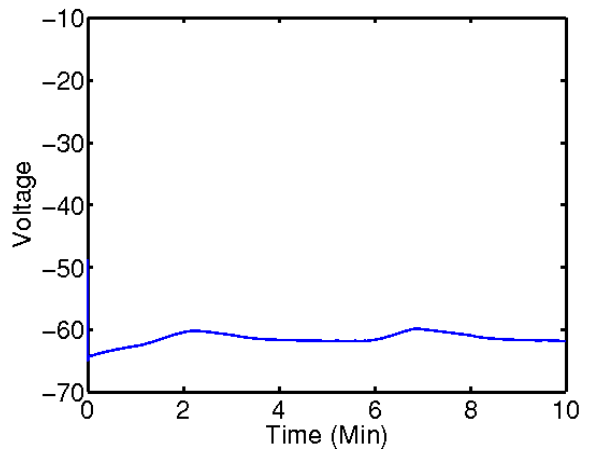

93 Mutated Cells

Figure 2: Voltage bursting as mutated cells are introduced into the $5 \times 5 \times 5$ islet. Coupling strengths kept constant at $g_{V}=75 \mathrm{pS}, g_{G 6 P}=0 \mathrm{~ms}^{-1}$, and $g_{F B P}=0 \mathrm{~ms}^{-1} . R_{\mathrm{GK}}$ is $0.2 \mathrm{~s}^{-1}$.

gradual loss of electrical bursting as the percentage of mutated cells in the islet increases. The behavior displayed corresponds to an islet coupled electrically but not metabolically; however, the behavior exhibited by islets with additional coupling parameters is analogous, which is analyzed in the next figure.

The studies in Figure 3 observe the number of electrical bursts over 10 minutes vs. the percentage of mutated cells in an $N \times N \times N$ islet with $N=5,6,7,8,9,10$. The cells are coupled electrically as well as metabolically $\left(g_{G 6 P}=0.035 \mathrm{~ms}^{-1}\right.$ and $\left.g_{F B P}=0.01 \mathrm{~ms}^{-1}\right)$. Figure 3 (a) gives the number of electrical bursts over 10 minutes vs. the percentage of mutated cells in a $5 \times 5 \times 5$ islet. Compare this to the top left plot in Figure 2; we see that the threshold of 93 of the 125 cells, or $74 \%$, is the same in both computational islets. The studies in Figure 3 also confirm that the bursting threshold is independent of islet size by running this simulation for larger values of $N$.

Table 1 lists the number of simulations in the studies for Figure 3 along with the shortest and longest runtime among the runs for each study, which cover a range due to the different numbers of mutated cells in each simulation. Since the runtime of each simulation and the number of simulations required for a study both increase as $N$ increases, the total runtime of studies for larger values of $N$ are very long. By using the distributed-memory cluster tara, described in Section 3.3, to run the simulations in the studies for larger $N$ simultaneously on several nodes, these studies become feasible in reasonable amount of time, when using the optimal memory-modified code with automatic differentiation, discussed in Section 3.2.

We conduct a study of the effect of $R_{\mathrm{GK}}$ values on oscillation death threshold in the islet with open channel mutations by varying the value of $R_{\mathrm{GK}}$ and documenting the number of mutated cells for which electrical oscillations disappear. In this study, we hold coupling constant at $g_{V}=100 \mathrm{pS}, g_{G 6 P}=0 \mathrm{~ms}^{-1}$, and $g_{F B P}=0 \mathrm{~ms}^{-1}$ and do not consider heterogeneity 


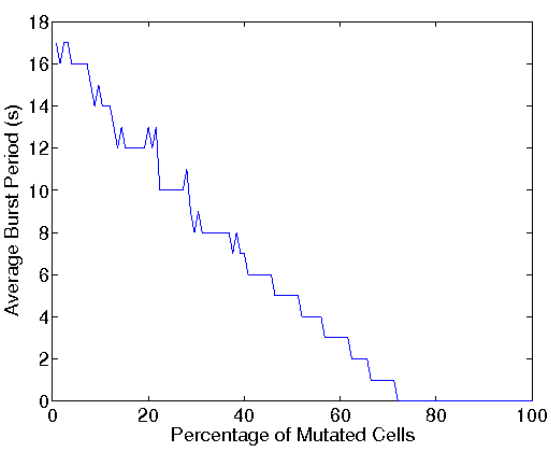

(a) $N=5$

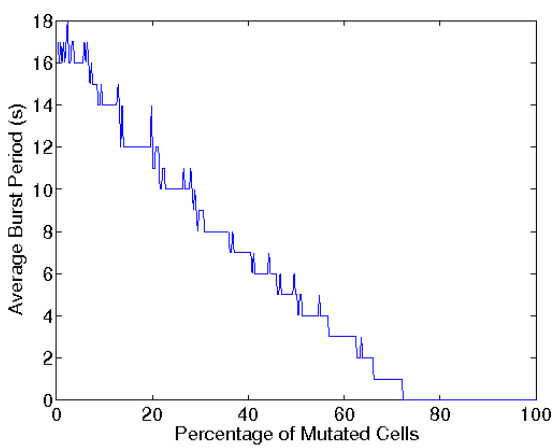

(c) $N=7$

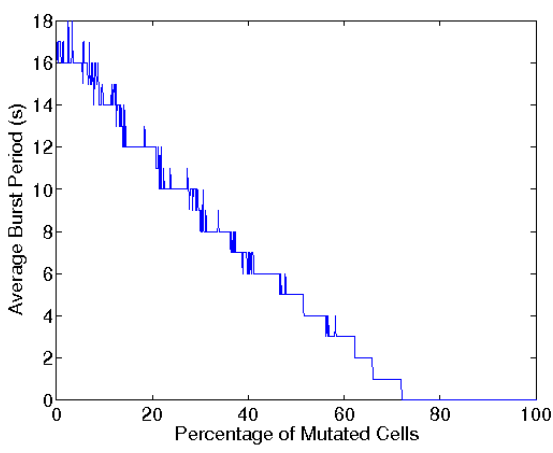

(e) $N=9$

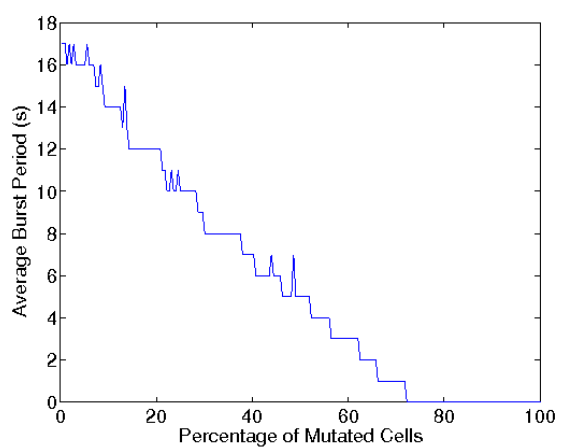

(b) $N=6$

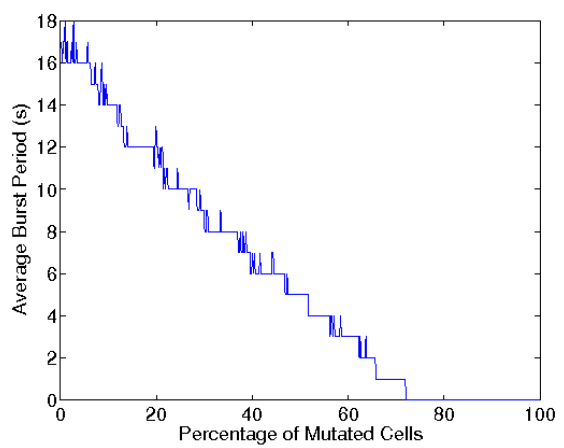

(d) $N=8$

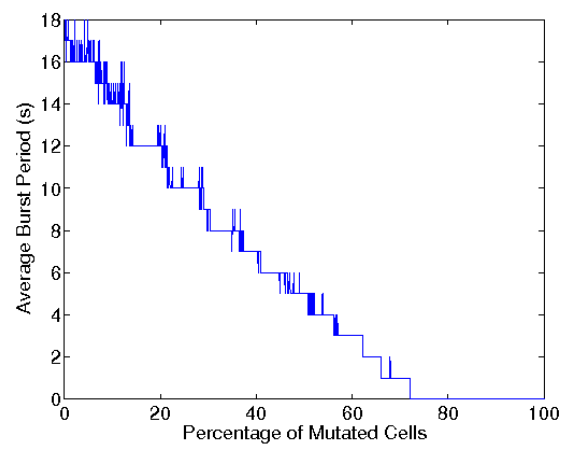

(f) $N=10$

Figure 3: Average burst period vs. percentage of mutated cells in an $N \times N \times N$ islet with $n=5,6,7,8,9,10$. Coupling strengths kept constant at $g_{V}=100 \mathrm{pS}, g_{G 6 P}=0.035 \mathrm{~ms}^{-1}$, and $g_{F B P}=0.01 \mathrm{~ms}^{-1}$. $R_{\mathrm{GK}}$ is $0.2 \mathrm{~s}^{-1}$.

Table 1: The number of runs, minimum runtime in HH:MM:SS, maximum runtime in HH:MM:SS for $600,000 \mathrm{~ms}$ with the range of islet dimensions $N \times N \times N$ considered in Figure 3 using the memory-modifed ode15s code.

\begin{tabular}{|c|c|c|c|}
\hline$N \times N \times N$ & Number of runs & Minimum time & Maximum time \\
\hline $5 \times 5 \times 5$ & 125 & $00: 00: 17$ & $00: 01: 54$ \\
$6 \times 6 \times 6$ & 216 & $00: 00: 27$ & $00: 03: 09$ \\
$7 \times 7 \times 7$ & 343 & $00: 00: 41$ & $00: 05: 00$ \\
$8 \times 8 \times 8$ & 512 & $00: 01: 10$ & $00: 08: 29$ \\
$9 \times 9 \times 9$ & 729 & $00: 01: 59$ & $00: 13: 56$ \\
$10 \times 10 \times 10$ & 1000 & $00: 03: 03$ & $00: 22: 22$ \\
\hline
\end{tabular}




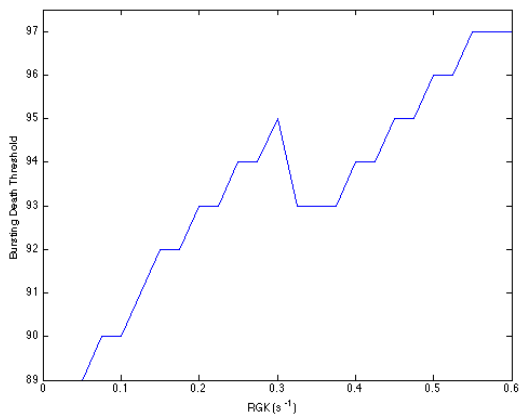

Figure 4: Bursting death threshold vs. $R_{\mathrm{GK}}$.

Table 2: Bursting death threshold vs. $R_{\mathrm{GK}}$.

\begin{tabular}{|c|c||c|c||c|c||c|c|}
\hline$R_{\mathrm{GK}}$ & Threshold & $R_{\mathrm{GK}}$ & Threshold & $R_{\mathrm{GK}}$ & Threshold & $R_{\mathrm{GK}}$ & Threshold \\
\hline 0.050 & 89 & 0.200 & 93 & 0.350 & 93 & 0.500 & 96 \\
0.075 & 90 & 0.225 & 93 & 0.375 & 93 & 0.525 & 96 \\
0.100 & 90 & 0.250 & 94 & 0.400 & 94 & 0.550 & 97 \\
0.125 & 91 & 0.275 & 94 & 0.425 & 94 & 0.575 & 97 \\
0.150 & 92 & 0.300 & 95 & 0.450 & 95 & 0.600 & 97 \\
0.175 & 92 & 0.325 & 93 & 0.475 & 95 & & \\
\hline
\end{tabular}

in the values of $R_{\mathrm{GK}}$ in the two cell types. Figure 4 and Table 2 contain the bursting death thresholds for multiple $R_{\mathrm{GK}}$ values in the $5 \times 5 \times 5$ islet.

We observe through repeated runs with mutated cells with varied parameters that the threshold depends on $R_{\mathrm{GK}}$ values in the islet but is independent of electrical coupling, at levels above those for synchronization where we can identify a single islet behavior, and of metabolic coupling strengths as well as of mutated cell arrangement within the islet. This threshold exists in every computational islet we considered in which cells with open channel mutations were introduced and increased.

\subsection{Numerical Study}

Efficiency studies were performed in [10] to show that the numerical implementation we use is optimized. We compare the original ode15s method with the memory-modified version to justify its continued use when we introduce metabolic coupling capabilities and associated computations into the model. Table 3 gives the wall clock time in HH:MM:SS for a simulation with coupling strengths of $g_{V}=75 \mathrm{pS}, g_{G 6 P}=0.01 \mathrm{~ms}^{-1}$, and $g_{F B P}=0.001 \mathrm{~ms}^{-1}$ for $200,000 \mathrm{~ms}$ in the grouped distribution. Table 1 contains runtimes for studies with final time of $600,000 \mathrm{~ms}$ for a range of percentage of mutated cells using memory-modified ode15s, hence with a range of times. Table 3 reports actual runtimes for a particular study with a final time of 200,000 ms for a fixed percentage of mutated cells using both original ode15s and memory-modified ode15s as prototype example, using the ADiMat software in both cases.

The results in Table 3 show significant improvement in simulation runtime, particularly as islet dimension $N$ increases. Islets in the human body have approximately 1,000 betacells, which makes the performance of simulations run at $N=10$ significant for biological applications. 
Table 3: Runtimes in HH:MM:SS for 200,000 ms with the range of islet dimensions $N \times N \times N$ considered in our studies comparing the original ode15s and the memory-modified ode $15 \mathrm{~s}$ code.

\begin{tabular}{|c|c|c|}
\hline$N \times N \times N$ & $\begin{array}{c}\text { Original } \\
\text { ode15s }\end{array}$ & $\begin{array}{c}\text { Memory-Modified } \\
\text { ode15s }\end{array}$ \\
\hline $3 \times 3 \times 3$ & $00: 00: 28$ & $00: 00: 21$ \\
$4 \times 4 \times 4$ & $00: 01: 38$ & $00: 00: 33$ \\
$5 \times 5 \times 5$ & $00: 04: 07$ & $00: 00: 46$ \\
$6 \times 6 \times 6$ & $00: 10: 02$ & $00: 01: 14$ \\
$7 \times 7 \times 7$ & $00: 24: 26$ & $00: 01: 45$ \\
$8 \times 8 \times 8$ & $00: 32: 52$ & $00: 02: 47$ \\
$9 \times 9 \times 9$ & $00: 48: 47$ & $00: 04: 51$ \\
$10 \times 10 \times 10$ & $01: 11: 58$ & $00: 07: 48$ \\
\hline
\end{tabular}

\section{Conclusions}

Through our studies, we gained a better understanding of the effects of coupling on the oscillation and bursting behaviors of pancreatic beta-cells.

When we ran simulations on islets containing cells with open $\mathrm{K}_{\mathrm{ATP}}$ channel mutations, there was a clear reduction in electrical bursts as higher proportions of mutated cells were implemented in our islet. Through simulating islets with large proportions of mutated cells, we were able to determine a bursting death threshold describing the least number of mutated cells necessary for electrical bursting to be lost. In the case of our $5 \times 5 \times 5$ islet model ran with one possible set of parameter values (Section 4.1), we found this threshold to be 93 of the 125 , or $74 \%$. This percentage, though independent of coupling strengths, mutated cell arrangement, and possibly islet dimension, is not independent of $R_{\mathrm{GK}}$ values. However, the bursting death threshold was between $71 \%$ and $78 \%$ for the range of $R_{\mathrm{GK}}$ tested (see Table 2) with less glucose flux requiring a slightly higher percentage of excitable beta-cells to maintain bursting. These simulations provide an interesting prediction for islets adapted with transgenic expression of mutated $\mathrm{K}_{\mathrm{ATP}}$ channels. Mutated islets that continue to burst should retain at least $29 \%$ excitable beta-cells, while those islets that do not burst would contain less than $22 \%$ excitable beta-cells. Furthermore, the bursting pattern may indicate the overall fraction of excitable cells with more dispersed spikes in a burst relating to fewer excitable cells and more dense bursting relating to greater numbers of excitable cells. We point out that it is somewhat remarkable in the model that up to $71 \%$ or greater of the $\mathrm{K}_{\mathrm{ATP}}$ channels can be dysfunctional yet an islet may retain the power to burst, which illustrates the robustness of bursting due to the high conductance of the $\mathrm{K}_{\mathrm{ATP}}$ channel in the beta-cells of the coupled islet.

Benninger et al. [2] looks at the interplay in multiple components in islets (e.g., gap junctional coupling, KATP channels, paracrine interaction) to maintain effective bursting response. Specifically they mutate KATP channels by replacing the ATP/ADP sensitive tail of the KATP channel leaving the functional potassium channel intact but no longer metabolically sensitive and possibly lower numbers of proteins inserted in the membrane [5]. They mutate the gap junctional proteins to nearly eliminate electrical coupling between islet cells. They show combinations of these mutations for increasing glucose levels and see the KATP mutant and wild type islets have the same glucose response curve for active islet cells. However, once uncoupled combining the gap junctional and KATP channel mutations approximately $70 \%$ of islet cells are active even in low glucose. This suggests this type of mutation is equivalent to the closed form of the KATP channel, which we have not studied, but a reduced KATP channel conductance model has been considered in a single cell from whole islet model and a cubic lattice of cells in [13].

However, in recent work Nguyen et al. 2014 [11] look at the gain-of-function mutation 
that expresses open KATP channels in mice. With open potassium channels imbedded in an affected beta-cell membrane, these beta-cells will tend to remain hyperpolarized. Nguyen et al. address the hypothesis that coupling within the islet, which silences any spontaneous bursting, might also silence normal bursting with the open channel mutation. They show that wild type (no mutations) have about $80 \%$ active cells in $20 \mathrm{mM}$ glucose, which drops to about $5 \%$ of normal cells when coupled to those in an islet with cells having the open channel mutations. Uncoupling the islet rescues the percentage of normal active cells to about $60 \%$. They measure about $50 \%$ mutated cells in an islet. In our model, to completely alleviate electrical bursting we find we need about $75 \%$. However, it is possible that one or two spikes per burst is insufficient to induce the experimentally measured activity. They also show that calcium level in the coupled mutated islet is about half that of the wild type islet suggesting some electrical activity is present in otherwise mostly ineffective islets. So our threshold of about $75 \%$ for complete loss of electrical bursting may be an overestimate for fraction of mutated cells required for down stream calcium and insulin secretion reductions.

\section{Acknowledgments}

These results were obtained as part of the REU Site: Interdisciplinary Program in High Performance Computing (www.umbc.edu/hpcreu) in the Department of Mathematics and Statistics at the University of Maryland, Baltimore County (UMBC) in Summer 2013, where they were originally reported in the tech. rep. [8]. This program is funded jointly by the National Science Foundation and the National Security Agency (NSF grant no. DMS-1156976), with additional support from UMBC, the Department of Mathematics and Statistics, the Center for Interdisciplinary Research and Consulting (CIRC), and the UMBC High Performance Computing Facility (HPCF). HPCF (www.umbc.edu/hpcf) is supported by the National Science Foundation through the MRI program (grant nos. CNS-0821258 and CNS-1228778) and the SCREMS program (grant no. DMS-0821311), with additional substantial support from UMBC. Co-author Jane Pan was supported, in part, by the UMBC National Security Agency (NSA) Scholars Program though a contract with the NSA. Graduate RA Samuel Khuvis was supported during Summer 2013 by UMBC.

\section{References}

[1] R. K. P. Benninger, W. S. Head, M. Zhang, L. S. Satin, and D. W. Piston, 2011. Gap junctions and other mechanisms of cell-cell communication regulate basal insulin secretion in the pancreatic islet. J. Physiol., 589(22), 5453-5466.

[2] R. K. P. Benninger, M. S. Remedi, W.S. Head, A. Ustione, D. W. Piston, and C. G. Nichols, 2011. Defects in beta cell $\mathrm{Ca}^{2+}$ signalling, glucose metabolism and insulin secretion in a murine model of $\mathrm{K}_{\mathrm{ATP}}$ channel-induced neonatal diabetes mellitus. Diabetologia, 54(5), 1087-1097.

[3] R. Bertram, L. Satin, M. Zhang, P. Smolen, and A. Sherman, 2004. Calcium and glycolysis mediate multiple bursting modes in pancreatic islets. Biophys. J., 87(5), 3074-3087.

[4] C. H. Bischof, H. M. Bücker, B. Lang, A. Rasch, and A. Vehreschild, 2002. Combining source transformation and operator overloading techniques to compute derivatives for MATLAB programs. In Proceedings of the Second IEEE International Workshop on Source Code Analysis and Manipulation (SCAM 2002), pages 65-72, Los Alamitos, CA, USA, 2002. IEEE Computer Society.

[5] E. A. Cartier, L. R. Conti, C. A. Vandenberg, and S.-L. Shyng, 2001. Defective trafficking and function of KATP channels caused by a sulfonylurea receptor 1 mutation 
associated with persistent hyperinsulinemic hypoglycemia of infancy. Proceedings of the National Academy of Sciences, 98(5), 2882-2887.

[6] Centers for Disease Control and Prevention. National diabetes fact sheet: national estimates and general information on diabetes and prediabetes in the United States, 2011. http://www.cdc.gov/diabetes/pubs/pdf/ndfs_2011.pdf, accessed December 10, 2013.

[7] S. Conde, T. Lebair, C. Raastad, V. Smith, K. Stern, D. Trott, M. K. Gobbert, B. E. Peercy, and A. Sherman, 2010. Enabling physiologically representative simulations of pancreatic beta cells. Technical Report HPCF-2010-21, UMBC High Performance Computing Facility, University of Maryland, Baltimore County.

[8] G. Gearhart, S. Jiang, T. J. May, J. Pan, S. Khuvis, M. K. Gobbert, B. E. Peercy, and A. Sherman, 2013. Investigating oscillation loss in computational islets. Technical Report HPCF-2013-14, UMBC High Performance Computing Facility, University of Maryland, Baltimore County.

[9] J. Keizer and G. Magnus, 1989. ATP-sensitive potassium channel and bursting in the pancreatic beta cell. a theoretical study. Biophys. J., 56(2), 229-242.

[10] S. Khuvis, M. K. Gobbert, and B. E. Peercy, 2013. Time-stepping techniques to enable the simulation of bursting behavior in a physiologically realistic computational islet. Submitted.

[11] L. M. Nguyen, M. Pozzoli, T. H. Hraha, and R. K. P. Benninger, 2014. Decreasing Cx36 gap junction coupling compensates for overactive KATP channels to restore insulin secretion and prevent hyperglycemia in a mouse model of neonatal diabetes. Diabetes.

[12] P. Smolen, 1995. A model for glycolytic oscillations based on skeletal muscle phosphofructokinase kinetics. J. Theor. Biol., 174(2), 137-148.

[13] K. Tsaneva-Atanasova and A. Sherman, 2009. Accounting for near-normal glucose sensitivity in Kir6.2[AAA] transgenic mice. Biophys. J., 97(9), 2409-2418.

[14] K. Tsaneva-Atanasova, C. L. Zimliki, R. Bertram, and A. Sherman, 2006. Diffusion of calcium and metabolites in pancreatic islets: Killing oscillations with a pitchfork. Biophys. J., 90(10), 3434-3446. 